\title{
Upcoming market catalysts in Q3 2017
}

Market catalysts in the third quarter of 2017 include top-line phase III trial results for several therapeutics: patisiran (developed by Alnylam Pharmaceuticals) for the treatment of hereditary ATTR amyloidosis with polyneuropathy (hATTR-PN); durvalumab (developed by AstraZeneca) for the first-line treatment of locally advanced or metastatic non-small-cell lung cancer (NSCLC); and brexanolone (developed by Sage Therapeutics) for the treatment of super-refractory status epilepticus (SRSE).

Alnylam expects top-line results from the phase III APOLLO trial of the intravenously injected RNA interference (RNAi) therapeutic patisiran for the treatment of hATTR-PN (also known as familial amyloidotic polyneuropathy), which is caused by mutations in the gene coding for transthyretin (TTR). Patisiran uses Arbutus Biopharma's second-generation lipid nanoparticle technology to deliver a small interfering RNA (siRNA) that targets TTR. Phase II results for patisiran showed that TTR knockdown was maintained above $80 \%$ for more than 9 months, and also showed a consistent decrease in the $\mathrm{mNIS}+7$ score (an evaluation of muscle weakness, sensory and autonomic function and nerve conductance) over 12 months, which is the primary end point for the APOLLO trial. Patisiran is the furthest advanced therapeutic in Alnylam's pipeline, following the discontinuation of revusiran, another TTR-targeted siRNA that used a different delivery technology $-\mathrm{N}$-acetyl-Dgalactosamine (GalNAc) conjugation. A phase III trial known as ENDEAVOUR to evaluate revusiran for the treatment of hereditary ATTR amyloidosis with cardiomyopathy (hATTR-CM) was halted in October 2016 owing to an imbalance of mortality in the revusiran arm compared with placebo. As a precaution, the APOLLO data monitoring committee met at the request of Alnylam, and recommended continuation of this trial without modification. If successful, patisiran could become not only Alnylam's first approved drug, but also the first approved RNAi therapeutic. However, it is in a tight race with lonis Pharmaceuticals' inotersen, an antisense therapeutic targeting TTR, to be the first approved drug for hATTR-PN in the United States.

AstraZeneca plans to announce top-line results from the phase III MYSTIC trial of durvalumab for the first-line treatment of patients with stage IV locally advanced or metastatic NSCLC. Durvalumab, which gained its first approval by the FDA as a monotherapy for advanced bladder cancer in May 2017, is a human monoclonal antibody directed against programmed cell death protein 1 (PD1) ligand 1 (PDL1), a key immune checkpoint target. The phase II results for durvalumab in NSCLC were in line with other immunotherapies targeting PD1 or PDL1 in terms of overall response rate. AstraZeneca is currently running multiple pivotal trials of durvalumab in different NSCLC treatment settings, including trials known as ARCTIC, PACIFIC and ATLANTIC, as well as MYSTIC. In May 2017, AstraZeneca announced initial results from the PACIFIC trial, which is testing durvalumab as a sequential therapy following treatment with chemotherapy plus radiation therapy in patients with stage III NSCLC, reporting that the primary end point of improving progression-free survival had been met in a planned interim analysis. However, the results of the MYSTIC trial, in which durvalumab is being tested both as a monotherapy and in combination with tremelimumab - which targets another immune checkpoint, cytotoxic T lymphocyte antigen 4 (CTLA4) - are anticipated to be even more important for the impact of durvalumab overall, given the large size and highly competitive nature of the first-line NSCLC market and given that the trial will provide an indication of the effectiveness of the combination with tremelimumab.

Finally, Sage anticipates top-line data from the pivotal phase III STATUS trial of brexanolone (also known as SAGE-547), an intravenously injected allosteric modulator of synaptic and extra-synaptic $\mathrm{GABA}_{A}$ receptors, in patients with SRSE (defined as recurrent seizure activity unresponsive to initial anaesthetic therapy). In a phase I/II trial , 8 of 11 patients evaluable for efficacy testing were able to be successfully weaned off their anaesthetic agents while brexanolone was being administered and 8 patients were weaned off brexanolone without recurrence of SRSE. Brexanolone was granted both Orphan Drug and Fast Track designation by the FDA in 2014, and after meeting with the FDA in 2015, Sage secured a Special Protocol Assessment (SPA) for the STATUS trial. Positive results from the trial could provide Sage with the basis to seek approval for the first drug to reach market to treat SRSE. Sonny Nghiem is at Informa, 3655 Nobel Drive, San Diego, California 92122, USA. Sonny.Nghiem@informa.com doi: $10.1038 /$ nrd.2017.117 Published online 16 Jun 2017; corrected online 21 Jul 2017

The author declares no competing interests. 
CORRIGENDUM

\section{Upcoming market catalysts in Q3 2017}

Sonny Nghiem

Nature Reviews Drug Discovery 16, 449 (2017)

There was an error in the description of the patient population for the PACIFIC trial of durvalumab. This has been corrected in the html and pdf versions. 